\section{Praxisbeitrag - Von der Geisteswissenschaft zur angewandten Forschung - Ein Erlebnisbericht mit und über Prof. Wolfgang G. Stock}

https://doi.org/10.1515/iwp-2019-2036

Der folgende Beitrag soll meine kleine „Reise“ vom Studium der Informationswissenschaft in Düsseldorf zu meinen inzwischen 12 Jahren am Fraunhofer Institut für naturwissenschaftlich-technische Trendanalysen INT in Euskirchen (und das als Geisteswissenschaftler) skizzieren und dabei die Rolle von Professor Stock beschreiben und entsprechend würdigen. Denn er hat mit seinem Lehrstuhl und mit seiner Art Studierende möglichst früh an die Pra- xis zu gewöhnen entscheidend dazu beigetragen, wie diese Reise verlaufen ist.

Der Anfang fällt auf den Beginn meines Studiums der Informationswissenschaft an der Heinrich-Heine-Universität, wo auch Professor Stock selbst sein Studium absolvierte. Neben Düsseldorf war er in Köln, Graz, München und weiteren Orten tätig, stets im Dienste seines Fachs. Meine erste Erinnerung an ihn ist seine schwarze Lederjacke, die er oft bei Vorlesungen trug und die Tatsache, dass er entgegen aller Regeln der Rhetorik bei seinen Vorträgen immer eine Hand in der Hose hatte. Manchmal beide. Nun ja...

Auffällig war, dass er für sein Fach brannte und das lichterloh. Entsprechend leidenschaftlich erlebte ich ihn in der Lehre. Besonders war auch sein Umgang mit kritischen Anmerkungen von Studierenden anderer Disziplinen, die er eloquent und unterhaltsam konterte und dabei stets darauf achtete, dass der „zweifelnde Thomas“ sich mitgenommen fühlte. Professor Stock, das war schnell klar, wollte seine Studierenden möglichst früh mit der 
praktischen Informationswissenschaft in Berührung bringen. Regelmäßig stellten Praktikerinnen und Praktiker verschiedenster Berufsfelder im Hörsaal die beruflichen Perspektiven für Studierende der Informationswissenschaft vor. Und: er machte die Erstellung wissenschaftlicher Artikel zu einem zentralen Bestandteil seiner Seminare. Thema meiner Gruppe in einem solchen Seminar war die „Usability von Navigationssystemen im E-Commerce und bei informativen Websites“. Dies untersuchten wir anhand so bekannter Seiten wie ebay und Amazon, aber auch für Kartoo.com, einer Metasuchmaschine, die eine damals neue visuelle Art von Interface nutzte.

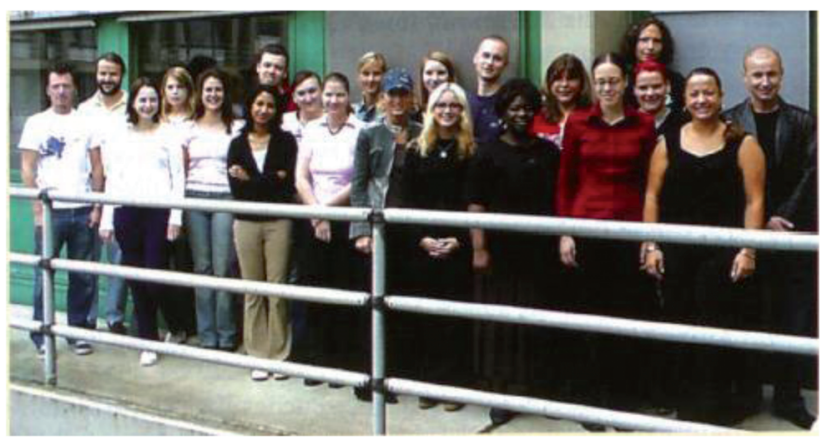

Abbildung 1: Die Mitglieder von E.V.I. Heine (der Autor des vorliegenden Artikels war an dem Tag verhindert). (Quelle: der im Beitrag erwähnte Artikel)

Dabei war die Art und Weise wie der Artikel zustande kam am wichtigsten. Professor Stock schaffte es einen Konsens innerhalb unseres Seminars herzustellen. So sollte bei der Masse von Autorinnen und Autoren (wir waren 30 Leute im Seminar) besser ein Synonym gefunden werden und so war prompt E.V.I. Heine (Evaluation von Informationsprodukten an der Heinrich-Heine-Universität Düsseldorf) geboren (s. Abb. 2). Meines Wissens war dieser Artikel der erste und gleichzeitig letzte, den E.V.I. Heine je veröffentlicht hat. Aber hier wurde die Basis für etwas gelegt, dass mich im Laufe meiner Karriere immer mehr beeindruckte: ich hatte meinen ersten wissenschaftlichen Artikel veröffentlicht ${ }^{1}$, ohne, dass das je mein Plan gewesen wäre. Und das Forschen, Schreiben und Publizieren hatte Spaß gemacht. Größtenteils.

So hängte ich nach meinem Studium auch noch eine Promotion in dem Fach an. Professor Stock war ein eher zurückhaltender Doktorvater im positiven Sinne. Er hatte
Zeit, wenn ich Fragen hatte oder Kooperationen anstoßen wollte, aber die Impulse mussten von mir kommen. Ein Arrangement, dass sehr gut zu mir passte und passt. Und so konnte ich meine Promotion erfolgreich abschließen und mir die beliebten zwei Buchstaben an meinen Namen heften.

Mit dem Beginn meiner Promotion fing ich auch parallel an, im Rahmen eines Lehrauftrags, ein Seminar zu meinem Promotionsthema durchzuführen. Dank meines Doktorvaters konnte ich diesem Lehrauftrag über zehn Jahre lang fast durchgängig nachkommen. So wird, denke ich, deutlich, dass ein Großteil des Rüstzeugs, um mich auch bei der Fraunhofer Gesellschaft, die sich die angewandte Forschung auf die Fahnen geschrieben hat, gut einbringen zu können, auf meinem Studium und meiner Promotion in Düsseldorf basiert.

Für viele ehemalige Studierende von Professor Stock endet mit seiner Pensionierung und dem Auslaufen der Studiengänge der Informationswissenschaft in Düsseldorf ein Stück persönlicher Geschichte. Aber: diejenigen, mit denen ich noch studiert oder die ich in meinen Lehrveranstaltungen kennengelernt habe, wurden, soweit ich das beurteilen kann, durch ihr Studium sehr gut auf ihre berufliche Zukunft vorbereitet. Sie sind in die Industrie gegangen (z.B. zu Google), der Forschung treu geblieben (z.B. als Professorinnen und Professoren) und mindestens einer ist aktuell Gruppenleiter bei Fraunhofer. Nicht schlecht und für eine Geisteswissenschaft nicht unbedingt typisch. Natürlich sind meine Schilderungen sehr individuell und zeigen nur einen kleinen Ausschnitt vom Wirken Professor Stocks, aber um mehr sollte es hier auch gar nicht gehen.

Und so bleibt mir eigentlich nur folgendes Fazit: Vielen Dank, lieber Wolf, für Deine Unterstützung und Dir viel Erfolg im verdienten Ruhestand und an der Uni Graz! Ich hoffe, man wird sich hier oder da dann doch nochmal begegnen!

Dr. Miloš Jovanović Euskirchen

1 Der Artikel ist online nicht verfügbar, aber eine Suche über Google Scholar führt immerhin zu den bibliographischen Angaben: E.V.I. Heine, (2003). Usability von Navigationssystemen im E-Commerce und bei informativen Websites - des Nutzers Odyssee. Information-Wissenschaft \& Praxis, 54, 405-414. 